EXEMPLARIa Classica

Journal of Classical Philology

21, 2017, pp. 63-72

ISSN 1699-3225

\title{
¿LAPSUS BIZANTINOS ELOCUENTES?: LA TRADICIÓN TEXTUAL DE CORNUTO Y EL COPISTA DEL CÓDICE $K^{1}$
}

\author{
José B. TORRES GUERRA \\ Universidad de Navarra \\ jtorres@unav.es
}

SUMMARY

This article analyses several lapsus calami attested in Cornutus's textual transmission. On the basis of the manuscripts themselves, these scribal mistakes cast light on the internal history of scribes and their world. The case of the Byzantine scholar who copied manuscript $\kappa$, from which a fourth part of the Cornutean manuscripts derives, is analysed in detail.

KEY WORDS

Cornutus's textual history; Greek scholars in the Renaissance; Scribal mistakes.
RESUMEN

Este estudio analiza varios lapsus calami atestiguados en la transmisión textual de Cornuto. Su interés consiste en que arrojan luz, a partir de los propios manuscritos, sobre la intrahistoria de la labor de copia y el mundo de los copistas. Se estudia en concreto el caso del copista bizantino del códice $\kappa$, del que deriva una cuarta parte de los manuscritos de Cornuto transmitidos.

\section{Palabras clave}

Errores de copia; Historia textual de Cornuto; Humanistas griegos del Renacimiento.

Fecha de recepción: 24/01/2017

Fecha de aceptación y versión final: 13/07/2017

${ }^{1}$ Deseo expresar mi agradecimiento a los profesores Sanz (Universidad de Extremadura) y Hernández (Universidad Complutense de Madrid) por la atención con que leyeron y discutieron conmigo versiones previas de este estudio. Cualquier error que pudiera contener es de mi exclusiva responsabilidad. 
El manual de crítica textual de West ${ }^{2}$ ejemplifica su exposición teórica con diversos pasajes griegos y latinos cuyas dificultades textuales discute. En Hes.

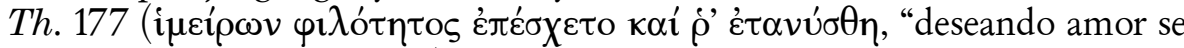
echó encima y se extendió") el correcto i $\mu \varepsilon$ íp v , "deseando", aparece en un códice (Arundel 522) como i $\mu \varepsilon i ́ p \omega$, "deseo". A propósito de ello comenta West: "possibly the scribe who wrote i $\mu \varepsilon i \rho \omega$, seeing the words "longing for sex', at once thought of his own longing”. En la historia de los textos se pueden encontrar numerosos ejemplos para los que cabe plantear como hipótesis que el subconsciente del copista pudo inducirlo a cometer errores parecidos al que detecta West en este caso. La cuestión, de interés para la crítica textual por cuanto atañe a la tipología de los errores de copia, ha sido estudiada en detalle, entre otros muchos críticos, por Havet o Timpanaro ${ }^{4}$.

Este estudio propone y discute algunos posibles errores similares, presentes

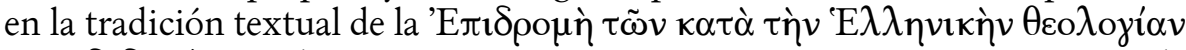
$\pi \alpha \rho \alpha \delta \varepsilon \delta$ $\mu \varepsilon ́ v \omega v$, el Repaso de las tradiciones teológicas de los griegos de Aneo Cornuto 5 . Como primer ejemplo se aduce una variante que aparece en un pasaje relativo a Ártemis; el ejemplo presenta cierta afinidad con el caso discutido por West:

Corn. ND 34 (71.19-72.2)

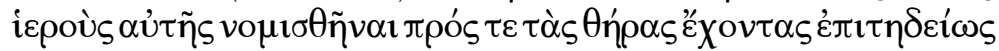

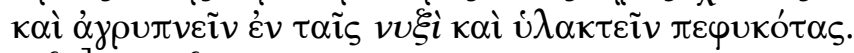

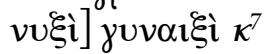

${ }^{2}$ Cf. M. West, Textual Criticism and Editorial Technique Applicable to Greek and Latin Texts, Stuttgart 1973.

${ }^{3}$ Cf. West, Textual Criticism, 107.

${ }^{4} \mathrm{La}$ obra de L. Havet (Manuel de critique verbale appliquée aux textes latins, Roma 1967 = Paris 1911) sigue siendo apreciada por su clasificación de los tipos de error y la abundancia de sus ejemplos. El libro de S. Timpanaro (Il lapsus freudiano. Psicanalisi e critica testuale, Firenze 1975), rico en observaciones de calado, está planteado como una crítica a la interpretación psicoanalítica del lapsus. Por supuesto son igualmente valiosas las aportaciones de A. Dain (Les manuscrits, Paris 1964²) o G. Pasquali (Storia della tradizione e critica del testo, Firenze $1988=1955^{2}$; cf. 483-6).

${ }^{5}$ Sobre Cornuto y su obra, cf. G. W. Most, “Cornutus and Stoic Allegoresis”, ANRW II 36.3, 1989, 2014-65; I. Ramelli, Anneo Cornuto. Compendio di teologia greca, Milano 2003; H. G. Nesselrath, ed., Cornutus. Die Griechischen Götter: ein Überblick über Namen, Bilder und Deutungen, Tübingen 2009. Hay una traducción al castellano del manual de Cornuto, al que se le da el título citado en el texto, en J. B. Torres Guerra, Mitógrafos griegos: Paléfato, Heráclito, Anónimo Vaticano, Eratóstenes, Cornuto, Madrid 2009.

${ }^{6}$ El texto de Cornuto se cita por la edición de Lang (C. Lang, Cornuti Theologiae Graecae Compendium, Leipzig 1881), indicando el capítulo de la obra y, entre paréntesis, las páginas y líneas de esa edición. Las palabras que plantean problemas textuales se editan en cursiva.

${ }^{7}$ Las observaciones sobre la transmisión textual de Cornuto se basan en una revisión personal de los códices, consultados de forma directa, en copias digitales u otros formatos, salvo indicación en sentido contrario. Las abreviaturas de códices, arquetipo, hiparquetipos y códices conjeturales son las de P. Krafft, Die handschriftliche Überlieferung von Cornutus' 
Concuerda con esto también el considerar que le están consagrados los perros, que están preparados para las cacerías y tienen la disposición natural de velar por las noches y ladrar ${ }^{8}$.

La variante juvaí̧i no está recogida en el aparato de la que es aún la edición de referencia de Cornuto, publicada por Lang en 18819; en cambio sí da cuenta de ella en nota la edición anterior de Osann (1844), quien indica, a propósito de $v \mathbf{v} \xi \grave{i}$ y en referencia a la edición Aldina de 1505: "Male Ald. $\gamma \cup v \alpha \mathbf{\imath}$ ì" $\kappa$, conjetural, del que derivan diez de los códices de Cornuto conservados según el análisis de Krafft ${ }^{11} \mathcal{\kappa}$ es copia de C (Vaticanus gr. 1314), compuesto hacia el año 1449 y derivado de $\varphi^{12}$; la fecha en que se pusiera por escrito $\kappa$ se debe situar en algún momento de la segunda mitad del s. XV, antes de la elaboración de las que deben de ser sus copias más antiguas, los códices $\mathrm{P}^{a}$ (Parisinus gr. 2860) y A (Monacensis gr. 536) ${ }^{13}$.

Desde un punto de vista textual no cabe duda de que la lectura correcta es $\nu$ u̇̀i. La cuestión es cómo se explica que el desconocido copista de $\kappa$

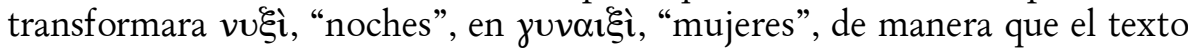
ya no hable de "velar en las noches" sino de "velar en las mujeres". A priori se podría pensar que el copista de $\kappa$ confundió la $v$ inicial de su modelo con una $\gamma(\boldsymbol{v} v \xi \grave{i} \rightarrow \gamma v \xi \xi i)$ y que después corrigió la forma intentando encontrarle un sentido ( $\gamma \cup \xi i \mathrm{i} \rightarrow \gamma u \nu \alpha \mathbf{\xi} \mathbf{i})$; pero, como se ha dicho, conocemos el modelo de $\kappa, C$, y la caligrafía de C hace muy improbable una confusión entre $v$ y $\gamma$

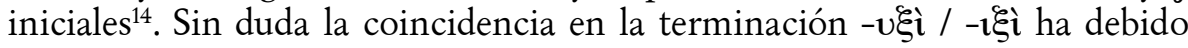

Theologia Graeca, Heidelberg 1975. Según este estudio, la tradición manuscrita de Cornuto deriva de un arquetipo medieval, $\omega$, y se divide en dos familias, $a$ y $\delta$; de $a$ derivan la familia $\lambda$ y los códices x y r; $\delta$, la rama más extensa de la tradición, incluye las familias $b, \varphi$ y el códice $\mathrm{m}$; sobre la filiación de $\kappa$, cf. lo que dice a continuación el cuerpo del artículo.

${ }^{8}$ Las traducciones reproducen con ligeras modificaciones las publicadas en Torres, Mitógrafos griegos.

${ }^{9}$ Cf. Lang, Cornuti Theologiae. Es preciso acudir aún a esta discutida edición a pesar de que distintos críticos anunciaron a lo largo del siglo XX su intención de preparar un nuevo texto crítico; cf. Krafft, Die handschriftliche Überlieferung, XI.

${ }^{10}$ Cf. F. Osann, L. Annaeus Cornutus de natura deorum ex schedis Johannis Bapt. Casp. d'Ansse de Villoison, Göttingen 1844, 207.

${ }^{11}$ Cf. Krafft, Die handschriftliche Überlieferung, 256-76. AB ${ }^{\mathrm{rfKL}} \mathrm{P}^{\mathrm{fa}} \mathrm{UV}^{\mathrm{b}} \mathrm{V} \mathrm{PW}$ son los códices conservados derivados de $\kappa$; la edición Aldina se basa en un códice perdido, $\mu$, apógrafo de $\kappa$; cf. Krafft, Die handschriftliche Überlieferung, 262-7. Los dos errores conjuntivos más significativos de $\kappa$ son la inclusión de una versión alternativa del capítulo sobre las Súplicas (12)

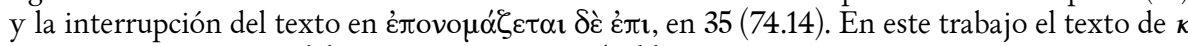
se reconstruye a partir del consenso entre KUV'Ald.

${ }^{12} \mathrm{Cf}$. Krafft, Die handschriftliche Überlieferung, 140.

${ }^{13} \mathrm{Cf}$. Krafft, Die handschriftliche Überlieferung, 121, 157.

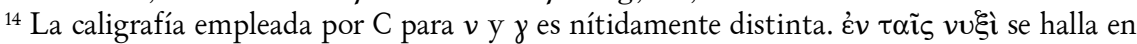
el f. 208v, 1. 23, de C. 


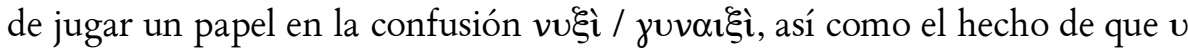
sea el núcleo de la primera sílaba en ambas palabras. Aun así, no parece que el error se pueda explicar solo apelando a la paleografía o la fonética ${ }^{15}$.

Este lapsus concreto que se introdujo en $\kappa$, y a partir de él en sus derivados y en la editio princeps del mitógrafo ${ }^{16}$, se podría explicar, como en el caso sugerido por West, apelando a inquietudes no conscientes del copista que

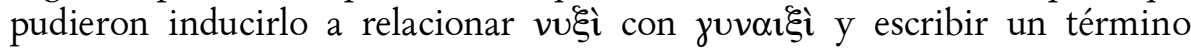
en lugar del otro. Es obvio que la hipótesis, aun siendo plausible, no es demostrable. No se dan las condiciones para situarla en un nivel distinto del de la conjetura sin conocer algo más sobre el copista de un códice perdido como $\kappa$. Pero saber algo más sobre ese amanuense solo se puede lograr, en todo caso, apelando al testimonio de otros errores que se puedan reconstruir para el códice conjetural que copió, esto es, intentando establecer una tipología de sus errores particulares ${ }^{17}$.

En este sentido se debe comentar de manera previa que la persona en cuestión tenía competencia suficiente como para enmendar errores de su modelo C. En las mismas líneas del capítulo 34 del Repaso antes citadas aparece la forma $\sigma u v \omega \delta \delta$ òv. En lugar de $\sigma u v \omega$ çòv, "que concierta con", "concordante", el hiparquetipo $\varphi$, compuesto como muy tarde en la primera

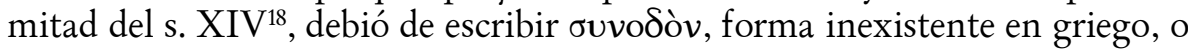

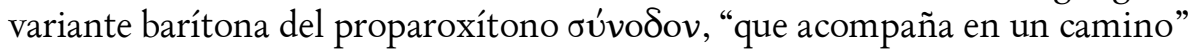
como adjetivo y, como sustantivo, "asamblea, reunión, sínodo"; el error se produjo por pérdida de la cantidad vocálica y confusión subsiguiente entre

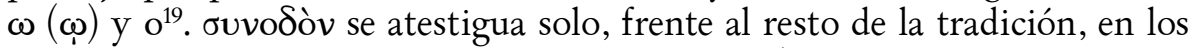
dos únicos derivados directos de $\varphi$, los códices Q (Laurentianus plut. 31 cod. 37) y $\mathrm{C}$, así como en todos los derivados de este último: a excepción de las copias que han de proceder de $\kappa$ según la crítica textual ${ }^{20}$. El copista de $\kappa$

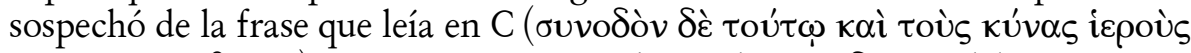

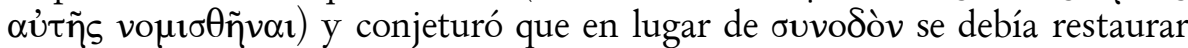
бuvœ人ò̀, la forma atestiguada en el resto de la tradición ${ }^{21}$.

${ }^{15}$ Como recuerdan Pasquali (Storia della tradizione, 471) y Timpanaro (Il lapsus freudiano, 24), son raros los lapsus que se explican solo por errores paleográficos.

${ }^{16}$ En la Aldina; cf. n. 11.

${ }^{17}$ Cf. Timpanaro, Il lapsus freudiano, 29-30.

${ }^{18}$ El terminus ante quem para la redacción de $\varphi$ lo proporciona su copia más antigua, Q; sobre la datación de este manuscrito (primera mitad del s. XIV), cf. Krafft, Die handschriftliche Überlieferung, 44 .

${ }^{19}$ Sobre la posibilidad de interpretar como error clerical la sustitución de $\sigma u v \omega$ cò̀ $v$ por

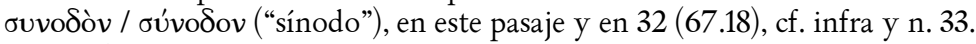

${ }^{20}$ Cf. n. 11.

${ }^{21}$ En $\lambda$ y $b$; los códices xrm, fragmentarios, no conservan este pasaje; cf. n. 7. A priori se podría suponer que $\kappa$ enmendó este lugar porque comparó el texto de $\mathrm{C}$ con un códice independiente de $\varphi$; pero tal posibilidad no es segura porque, si hubiera consultado otro códice,

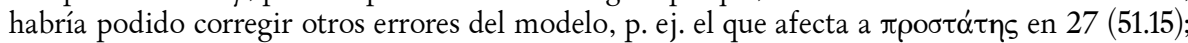


La competencia lingüística del copista de $\kappa$ también la indica el hecho de que corrigiera también con acierto el texto que presenta su modelo $\mathrm{C}$ poco

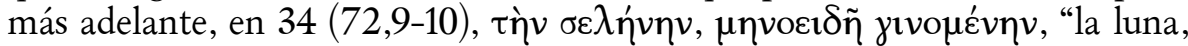
en fase creciente". $b^{22}$ y C escribieron por iotacismo, en lugar de $\mu \eta \eta$ voєı $\delta \tilde{\eta}$, $\mu \varepsilon ı v o \varepsilon \imath \delta \tilde{\eta}$. Esta forma fue enmendada por $\kappa$, quien restituyó $\mu \eta v o \varepsilon ı \delta \tilde{\eta}$ al igual que la segunda mano de B (Laurentianus plut. $60 \mathrm{cod}$. 19), códice que procede de $b$; en cambio, $\mathrm{G}$ (Baroccianus gr. 125), procedente también de $b$, advirtió el error pero lo corrigió de forma equivocada al sustituir $\mu \varepsilon ı v o \varepsilon \imath \delta \tilde{\eta}$ por $\mu$ ovozi $\delta \tilde{\eta}^{23}$.

Estos no son los únicos lugares en los que $\kappa$ intentó corregir fallos de C. Sin embargo, no siempre acertó en sus conjeturas. Se debe llamar la atención sobre

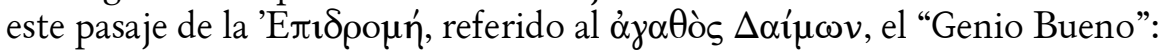

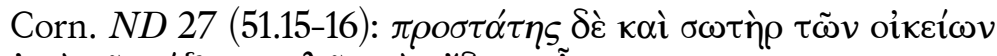

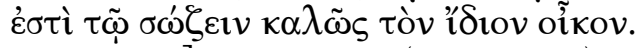

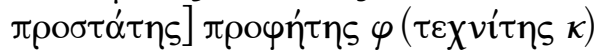

Es quien está al cargo y preserva las cosas de la casa debido a que preserva bien su propia casa.

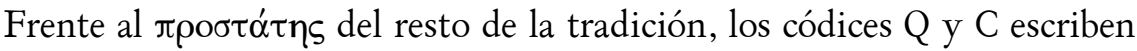
$\pi \rho \circ \eta^{\prime} \tau \eta$, forma que se debe retrotraer al hiparquetipo $\varphi$. Es obvio que la mención de un $\Delta \alpha i ́ \mu \omega v$ "profeta" carece de sentido en este pasaje. Lo más probable es que el amanuense de $\varphi$, cuando iba a copiar $\pi \rho o \sigma \tau \alpha \dot{\tau} \tau \eta$, tuviera ya a la vista el siguiente sustantivo, $\sigma \omega \tau \eta \dot{\rho} \rho$, "salvador". Una asociación de ideas que podría caracterizarlo como individuo perteneciente al ámbito eclesiástico, más el hecho de que las dos palabras compartieran prefijo y sufijo, lo llevó a

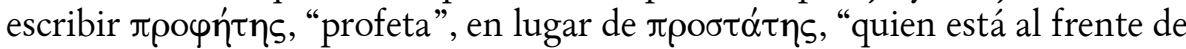
algo" ${ }^{24}$. $\kappa$, al copiar lo que leía en C, debió de advertir la inverosimilitud de que un texto de mitología hablase de un $\dot{\alpha} \gamma \alpha \theta$ òs $\Delta \alpha i \mu \omega v$ que era profeta y salvador; por ello conjeturó una forma alternativa relativamente adecuada al contexto, manteniendo el sufijo de agente: $\tau \varepsilon \chi v i$ in $\varsigma$, "artesano", variante de la que no se hace eco la edición de Lang aunque sí la incluye en nota el texto de Osann ${ }^{25}$.

cf. infra.

${ }^{22}$ Cf. n. 7.

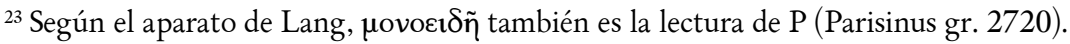

${ }^{24}$ Sobre los lapsus regresivos y su mayor frecuencia frente a los progresivos, cf. Timpanaro, Il lapsus freudiano, 104-5. Sobre la posibilidad de que el error sea propio de un eclesiástico, cf. lo que comenta West (Textual Criticism, 21) sobre la sustitución de $\kappa \alpha \theta \mathrm{o} \lambda \kappa \eta \dot{v}$ ("arrastre de

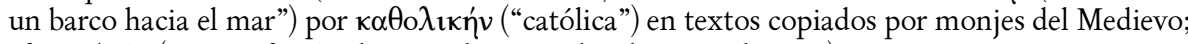
cf. también (con profusión de ejemplos tomados de textos latinos) Havet, Manuel de critique verbale, $263-4$.

${ }^{25}$ Cf. Osann, L. Annaeus Cornutus, 154. Lang tampoco recoge $\pi \rho \circ \varphi_{\eta} \tau \eta$ s. La variante $\tau \varepsilon$ Xíins no aparece, por cierto, como texto alternativo en C (cf. f. 203r, 1. 24). 
Junto a emendationes, acertadas o no, el copista de $\kappa$ también introdujo en su códice lecturas erróneas exclusivas de ese manuscrito perdido, los lapsus calami a los que concede atención especial este trabajo: recuérdese lo

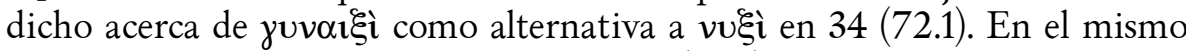
sentido se debe decir que en el capítulo 9 (9.12) los códices derivados de $\kappa$

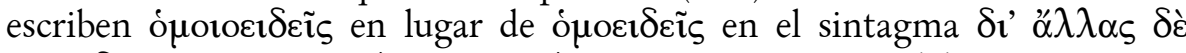
ó fáciles de reconocer"; aunque el error evoque la antigua discusión sobre la naturaleza de Dios Hijo, ónoov́rıs o ópotov́øıs en su relación con Dios Padre, resulta incierto proponer conjeturas de tal calado a partir de este lapsus aislado que puede obedecer a una simple confusión entre los primeros términos del compuesto, ó $\mu \mathrm{o}-\mathrm{y}$ ó $\mathrm{ot}^{26}$. Por otra parte todos los códices

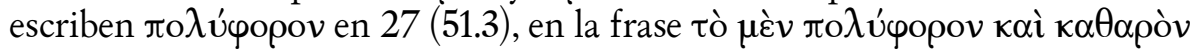
$\alpha i \stackrel{\alpha}{\mu} \pi \varepsilon \lambda$ oı $\pi \alpha \rho \imath \sigma \tau \tilde{\alpha} \sigma \iota$, "las viñas hacen presente la riqueza de frutos y la pureza" ${ }^{27}$; en cambio los códices que proceden de $\kappa$ cambian $\pi \mathrm{o} \lambda$ ú $\varphi \rho \rho o v$,

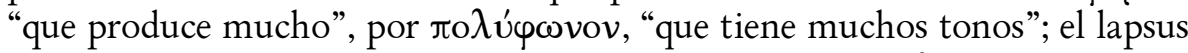
es llamativo por lo inadecuado que parece el adjetivo $\pi \mathrm{o} \lambda$ ú $\varphi \omega v o s$ en este contexto; la edición de Lang no hace mención de la variante aunque sí lo hace en nota la de Osann ${ }^{28}$. En el capítulo sobre Heracles, en 31 (64.17), el texto

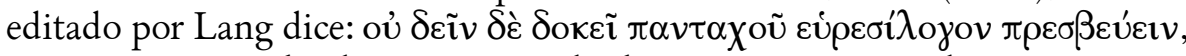
"parece que no se ha de venerar a todas horas a un inventor de argumentos"; $\kappa$ cambió $\pi \rho \varepsilon \sigma \beta \varepsilon v ́ \varepsilon ı v$ por $\pi \rho \varepsilon \sigma \beta u ́ \tau \eta \nu$, de forma que su texto pasó a referirse a un "anciano inventor de argumentos" del que no hay necesidad; tampoco resulta fácil encontrar una explicación para este cambio, que quizá no sea un error sino una emendatio con la que el copista quiso corregir un texto que le parecía sospechoso ${ }^{29}$.

Este repaso de los errores propios de $\kappa$ deja para el último lugar el curioso caso de un lapsus reincidente que afecta a tres lugares del Repaso. En 16

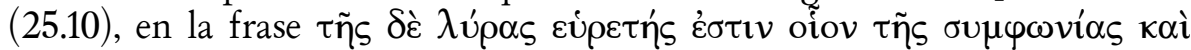
ónoגoyías ("Es inventor de la lira, como de la armonía y el acuerdo"), $\kappa$ debió de escribir ónovoías, "unidad de pensamiento", "concordia”, en lugar de ó $\mu$ o $\lambda_{\text {oxías; }}$ esta variante, a la que se refiere en nota Osann, no aparece en el aparato de Lang ${ }^{30}$. Se puede suponer que este es un lapsus aislado favorecido

\footnotetext{
${ }^{26}$ La cuestión del ónoov́øı del primer milenio a partir de la disputa arriana. Nótese que, al escribir ó $\mu$ oıøı $\delta$ ńs, el copista de $\kappa$ eligió un adjetivo poco frecuente en comparación con ó $\mu$ oвı $\delta$ '́s; cf. $L S J$, s. u.

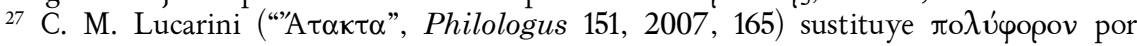

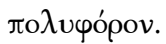

${ }^{28}$ Cf. Osann, L. Annaeus Cornutus, 154.

${ }^{29} \mathrm{La}$ caligrafía de C en este punto del manuscrito (f. 207r, 1. 12) es nítida. Sobre los problemas del pasaje, cf. Ramelli, Anneo Cornuto, 396. U. von Wilamowitz-Moellendorff (Coniectanea,

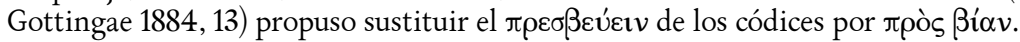

${ }^{30}$ Cf. Osann, L. Annaeus Cornutus, 74.
} 


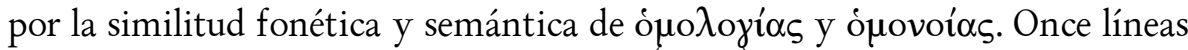
más adelante según el texto de Lang, en 16 (25.21), el códice $\kappa$ cometió un error

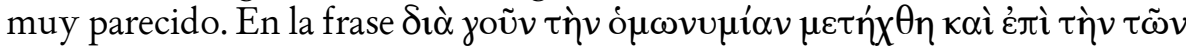

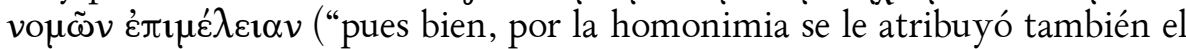
cuidado de las tierras de pasto") los códices derivados de $\kappa$ vuelven a escribir ó $\mu$ óvor $\alpha \nu$ en lugar de ó $\mu \omega v v \mu i ́ \alpha v$; en este nuevo caso la similitud fonética no es tan obvia y la semántica se limita al primer término del compuesto; Lang tampoco da cuenta de esta variante que Osann menciona en nota ${ }^{31}$. El mismo

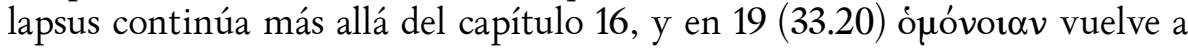
aparecer en los derivados de $\kappa$ en lugar de la lectura correcta, ahora ö $\mu$ or $\alpha \nu$,

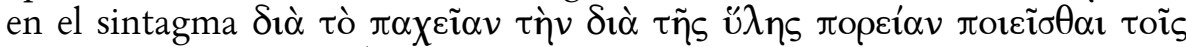

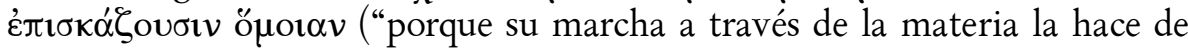
manera torpe, a semejanza de los que claudican”); Lang tampoco incluyó esta lectura en su aparato crítico aunque Osann sí lo había hecho en nota ${ }^{32}$.

Es llamativo que el copista se equivoque en lugares diferentes del Repaso ante tres palabras distintas que empiezan por ó $\mu \mathrm{o}(\omega)$ - y que sustituya las tres por un mismo sustantivo. Ello despierta la sospechosa de que la querencia de este copista por la ó $\mu$ óvor $\alpha$ puede no ser casual y sin duda es legítimo preguntarse por sus motivos. Sin embargo, como sucedió al principio con la sustitución de $v \mathbf{v} \xi \grave{i}$ por $\gamma u v \alpha \mathbf{\xi}$ ì , es muy incierto formular cualquier hipótesis sobre las razones por las que un copista de la segunda mitad del s. XV podía sentir preferencia por la palabra que en griego indica "concordia". A no ser que, hipotéticamente, cupiera establecer una relación entre todos o varios de estos lapsus.

Hasta el momento se ha comprobado que el desconocido copista de $\kappa$ tenía competencia suficiente en griego como para enmendar con acierto errores de sentido o gramaticales de su modelo según se ha visto a propósito de

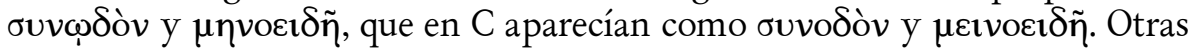
veces identificaba el error pero no acertaba en su solución como le ocurrió con $\tau \varepsilon X v i ́ \tau \eta \varsigma$, falso sustituto del $\pi \rho \circ \varphi \eta^{\prime} \tau \eta_{\varsigma}$ heredado por $\mathrm{C}$ del hiparquetipo $\varphi$. A su vez incluyó en su copia errores reproducidos luego en sus apógrafos:

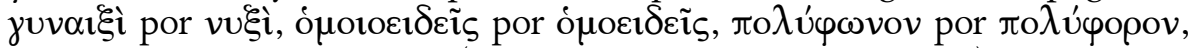

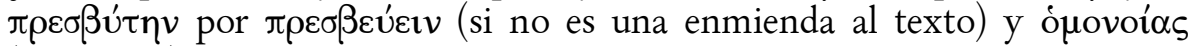

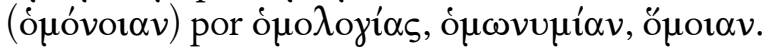

¿Pueden estos datos arrojar luz sobre la personalidad del copista de $\mathcal{\kappa}$, según se planteó de forma ideal al comienzo de este artículo? Por sí mismos los datos parecen indicar que, con mucha probabilidad, este copista de la segunda mitad del s. XV debía de tener el griego como lengua materna según sugiere su competencia lingüística. Otros copistas que han intervenido en el texto de Cornuto han dejado huellas que indican su probable pertenencia

${ }^{31}$ Cf. Osann, L. Annaeus Cornutus, 76.

${ }^{32}$ Cf. Osann, L. Annaeus Cornutus, 76. 
a ambientes eclesiásticos según se ha visto, para $\varphi$, en el posible caso de $\pi \rho \circ \varphi$ in en el Repaso $\sigma u v \omega \delta$ òv por $\sigma u ́ v o \delta o v^{33}$; eventualmente podría ser también un

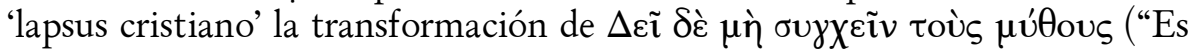

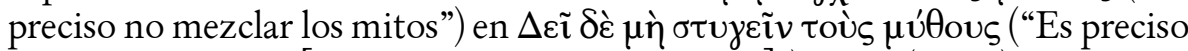
no odiar los mitos [sc., de la Antigüedad pagana]") en 17 (27.19), lapsus que se retrotrae por lo menos al arquetipo medieval $\omega^{34}$. En cambio, ninguna intervención del autor de $\mathcal{\kappa}$ sugiere que este fuera un eclesiástico; podría haberlo sido pero sus lapsus propios no ofrecen ningún dato positivo en este sentido salvo que se quiera llevar la argumentación al extremo y reconocer

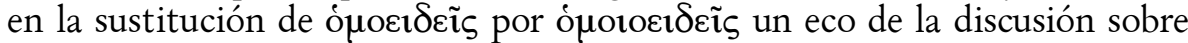

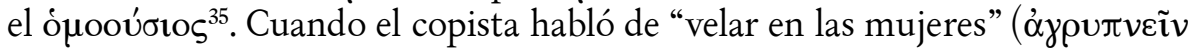

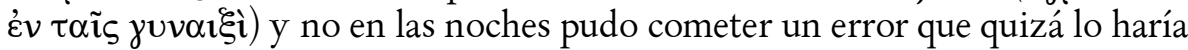
sonrojar a posteriori; pero este error solo parece indicarnos que el copista era varón, como seguramente era previsible. La situación puede ser distinta en el caso de otros errores, no en lo que se refiere al cambio de $\pi \rho \varepsilon \sigma \beta u ́ \tau \eta \nu$ por

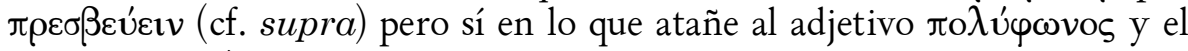
sustantivo ó $\mu$ óvor $\alpha$.

Desde un principio se ha hablado en este trabajo del copista de $\kappa$ como de una figura de identidad desconocida. Desde luego ello es así, aunque con matices. No se sabe nada de él pero sí de quien copió su modelo, el manuscrito $\mathrm{C}$, y de los autores de las otras cuatro copias derivadas directamente de C, las que se conservan a diferencia de $\kappa$ : los códices $\mathrm{H}$ (Matritensis 4628), $\mathrm{L}^{\mathrm{a}}$ (Laurentianus plut. 58 cod. 13), Ve (Venetus gr. 770) e Y (Venetus gr. 924) ${ }^{36}$. Estos cinco amanuenses presentan una serie de rasgos comunes que quizá se puedan atribuir también al responsable de la copia perdida.

${ }^{33}$ En $32(67,18)$ y en $34(71,19)$. Sobre las características del segundo caso y su aparición

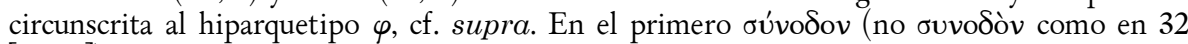
$[67,18])$ es la lectura casi unánime de los códices y se retrotrae al menos al arquetipo medieval $\omega$, sobre cuya fecha aproximada de composición cf. Krafft Die handschriftliche Überlieferung,

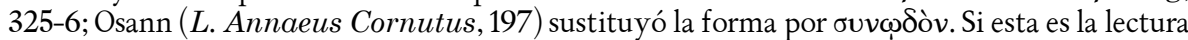
correcta en ambos casos, el cambio de una forma por otra pudo no producirse solo por la pérdida de la cantidad vocálica: es probable, aun sin poderse demostrar, que el copista medieval de $\omega$ y

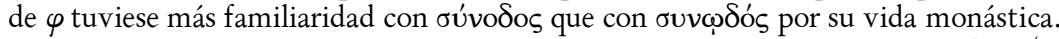

${ }^{34}$ El lapsus puede implicar una mezcla de factores ideológicos y paleográficos (cf. n. 15): un

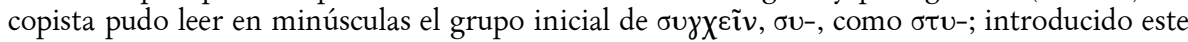

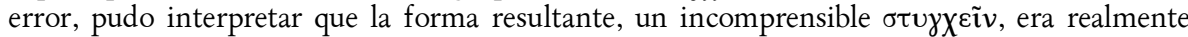

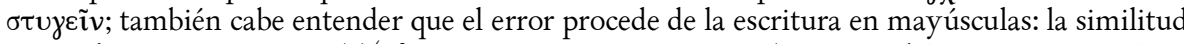
que se da en esta entre Y y T (cf. West, Textual Criticism, 25; A. Bernabé, Manual de crítica textual y edición de textos griegos, Madrid $\left.2010^{2}, 27\right)$ pudo dar pie a la confusión. бuðXعĩv es conjetura de Osann (L. Annaeus Cornutus, 81).

${ }^{35}$ Cf. supra y n. 26.

${ }^{36}$ Sobre $\mathrm{HL}^{\mathrm{a}} \mathrm{V}^{\mathrm{Y}} \mathrm{Y}$ como apógrafos de C, cf. Krafft, Die handschriftliche Überlieferung, 278-83, 283-300. 
Se sabe con seguridad que C fue copiado hacia 1449 (cf. supra) por Andronico Calisto y que este no utilizó un códice distinto de su modelo para la revisión del texto de Cornuto ${ }^{37}$. Andronico Calisto nació en el Imperio de Oriente y, a principios del s. XV, debió de trasladarse a Italia con ocasión del llamado concilio de Florencia, en el que se discutió sobre la unidad de las Iglesias de Oriente y Occidente ${ }^{38}$. Se sabe que formaba parte del entorno de una figura que desempeñó un papel clave en ese concilio, el cardenal Besarión, a cuyo círculo estuvieron vinculados, de una forma u otra, todos los copistas que reprodujeron el Repaso a partir de C, manuscrito que debió de permanecer en poder de Andronico Calisto hasta $1476^{39}$ : Juan Roso, que confeccionó el manuscrito La; Miguel Apostolio, autor del códice Ve; Demetrio Triboles, el copista de Y; y un colaborador desconocido de Constantino Láscaris, responsable de la copia de $\mathrm{H}^{40}$. Se trataba de humanistas griegos asentados en Italia desde mediados del s. XV, exiliados del mundo ortodoxo bizantino que trabajaron en un nuevo ambiente cultural, a menudo al amparo de Besarión y la Iglesia de Roma ${ }^{41}$. Es muy probable que el copista ignoto de $\kappa$ compartiera estos rasgos con ellos. En tal caso, cabe avanzar en la interpretación de esos dos lapsus extraños que insertó en su copia de Cornuto: $\pi$ o $\lambda u ́ \varphi \omega v o s$ y ó $\mu$ óvot $\alpha$.

En el contexto delineado en el párrafo anterior $\pi$ o $\lambda$ ú $\varphi \omega v o s$ podría reflejar la sugestión ejercida por la música polifónica de Occidente en un individuo criado y educado en la tradición musical bizantina, distinta de la occidental. Ante ese mismo trasfondo cultural no resulta tan hipotético sugerir una explicación para la recurrencia del lapsus ó $\mu$ óvor $\alpha$, "concordia", en $\kappa$ : este error repetido podría reflejar la aspiración del copista a la concordia entre Occidente y Oriente, entre las iglesias de Roma y Constantinopla, y su deseo de que tal acuerdo empujara a las naciones católicas a liberar su patria del poder del sultán ${ }^{42}$. Es cierto que tal hipótesis, aunque plausible, se resiste

${ }^{37}$ Cf. Krafft, Die handschriftliche Überlieferung, 139-40.

${ }^{38}$ Sobre Andronico Calisto, cf. A. Perosa, "Inediti di Andronico Callisto", Rinascimento 4, 1953, 3-15; J. Monfasani, Byzantine Scholars in Renaissance Italy: Cardinal Bessarion and other émigrés: Selected Essays, Aldershot 1995, XII, 396-7. El concilio, iniciado en Basilea en 1431, se trasladó sucesivamente a Ferrara en 1438 y Florencia en 1439; sus últimas decisiones se tomaron en Roma en 1445.

${ }^{39}$ En 1476 vendió sus manuscritos a Giorgio Valla; cf. Krafft, Die handschriftliche Überlieferung, 141.

${ }^{40} \mathrm{Cf}$. Krafft, Die handschriftliche Überlieferung, 55-60, 83-7, 160-2, 168-70. Para el caso de H, cf. también J. M. Fernández Pomar, "La colección de Uceda y los manuscritos griegos de Constantino Láscaris", Emerita 34, 1966, 211-88.

${ }^{41}$ Cf. J. Harris, Greek Emigres in the West, 1400-1520, Camberley 1995; Monfasani, Byzantine Scholars; N.G. Wilson, From Byzantium to Italy, Greek Studies in the Italian Renaissance, Baltimore 1992. Sobre copistas anónimos pertenecientes al círculo de Besarión, cf. T. Martínez Manzano, "Un copista del lustro boloñés de Besarión: el Anonymus LY”, Nع́a 'Pó $\mu \eta$ 10, 2013, 211-43.

${ }^{42}$ Se ha de recordar que el papa Sixto IV intentó en efecto organizar una cruzada contra Mehmed II en 1472. Durante los preparativos de esta empresa el cardenal Besarión acudió por 
a cualquier demostración en tanto que implica bucear en la conciencia de alguien muerto hace más de quinientos años sobre el que se dispone de un cuerpo tan reducido de datos. Aun así, podría no ser una casualidad sino el síntoma de una aspiración compartida que la versión griega del Laetentur caeli, la Bula de unión de las Iglesias emanada del concilio de Florencia (6 de julio de 1439), repita también por dos veces en sus primeras líneas la palabra ópóvota $\alpha^{43}$.

encargo papal ante el rey Luis XI de Francia para recabar su apoyo; al regreso de su misión falleció en Rávena (1472).

43 Cf. I. Gill, ed., Quae supersunt Actorum Graecorum Concilii Florentini necnon descriptionis cuiusdam eiusdem. II. Res Florentiae gestae, Roma 1953, 459:

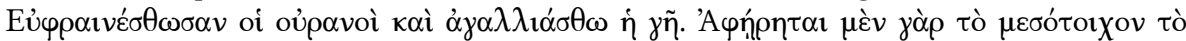

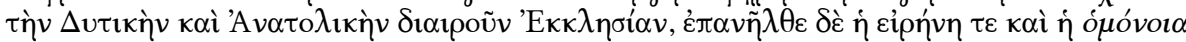

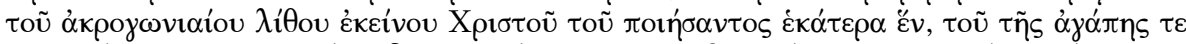

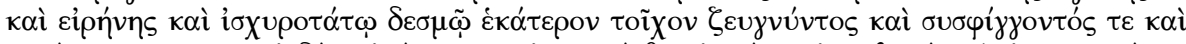

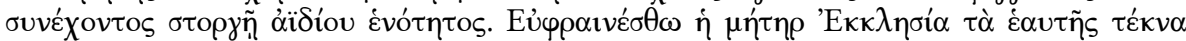

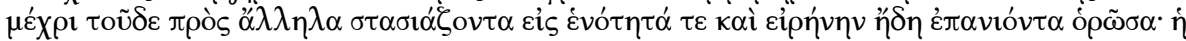

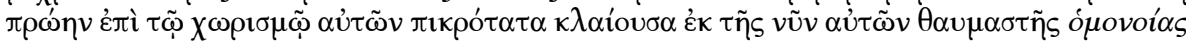

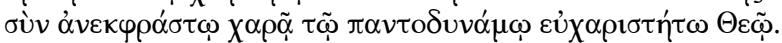

